\title{
ILLUMINATION NORMALIZATION BASED ON SIMPLIFIED LOCAL BINARY PATTERNS FOR A FACE VERIFICATION SYSTEM
}

\author{
Qian Tao, Raymond Veldhuis \\ Signals and Systems Group, Faculty of EEMCS \\ University of Twente, the Netherlands
}

\begin{abstract}
Illumination normalization is a very important step in face recognition. In this paper we propose a simple implementation of Local Binary Patterns, which effectively reduces the variability caused by illumination changes. In combination with a likelihood ratio classifier, this illumination normalization method achieves very good recognition performance, with respect to both discrimination and generalization. A user verification system using this method has been successfully implemented on a mobile platform.
\end{abstract}

\section{INTRODUCTION}

Nowadays biometric verification is widely used in various security applications such as secure access to a transaction or a network, and identity check at an airport. The larger context of our work is biometric authentication as a link between a user and a private PN (personal network), via an intermediate MPD (mobile personal device) [1]. For such a link, three major requirements are put forward, namely, security for the network, convenience to the user, and computational complexity for the device.

Face is one of the most important biometrics in use. It is convenient because little user effort is required for biometric collection; it is inexpensive because low-cost webcams suffice in most cases. Face verification is essentially a yes-or-no problem: to judge whether the claimed identity is correct or not by verifying the claimant's face image. Generally speaking, such a face verification task consists of two key stages: the feature vector extraction from the raw face image, and the classification of the feature vector. In both stages there are many challenges, such as face detection, face registration, illumination normalization, modeling and learning.

In this paper, we will address the illumination normalization in particular. Illumination is a very important problem in face recognition. Research has shown that for a face image, the variability caused by illumination changes even exceeds the variability caused by identity changes [3]. As an example, Fig. 1 shows the face images of the same subject under two lands.

This work is funded by the Freeband PNP2008 project of the Nether-
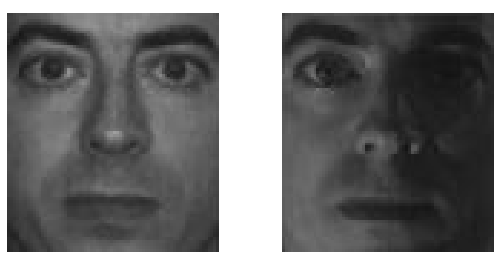

Fig. 1. Examples of the same subject seen under different illuminations [2].

different illuminations from Yale Face Database B [2]. Such illuminations will largely influence the performance of face recognition. This illumination problem can be considered in the classification stage, such as in [4], but then the classifier has to incorporate a model of the illumination variability. In this paper we try to solve the illumination problem during the feature extraction stage, and keep the simplicity of the classifier. For this purpose we preprocess the face image based on a simplified implementation of Local Binary Patterns (LBP) [5], which effectively reduces the variability caused by illumination changes. In combination with a likelihood ratio classifier, very good recognition performance is achieved, with respect to both discrimination and generalization. A verification system using this method has been successfully implemented on a mobile platform.

This paper is organized as follows. Section 2 reviews the existent illumination normalization methods; Section 3 provides our solution for illumination normalization; Section 4 describes the verification algorithm; Section 5 shows the experimental results; and Section 6 gives the conclusions.

\section{REVIEW OF ILLUMINATION NORMALIZATION METHODS}

Two categories of illumination-normalization approaches can be distinguished. The first is to investigate the physical model, including the imaging mechanism and its interactions with the $3 \mathrm{D}$ object surface, such as the work on the illumination cone [6], spherical harmonics [7] and illumination light-fields [8]. These methods have theoretical grounds and achieve good 


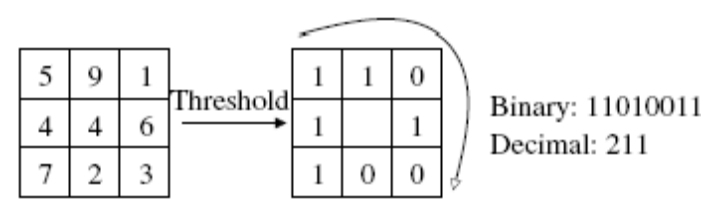

Fig. 2. The LBP operator: the binary result and the decimal result [5].

performance, but they generally require training data under different illuminations, and have a high computational complexity.

The second approach works on the $2 \mathrm{D}$ image itself and tries to normalize the illumination by preprocessing the pixelvalues. Examples are the Retinex approach [9] [10], quotient image [11] [12], diffusion approach [13] [14], and LBP preprocessing [15]. The second approach is direct and simple and often an underlying imaging model can be identified, providing a theoretical foundation. In our verification application, the mobile device has a low-resolution camera, and limited computational resources. Besides, enrolment of a user under many illumination conditions is not possible. Therefore, we adopt the latter preprocessing approach.

\section{ILLUMINATION NORMALIZATION BASED ON SIMPLIFIED LBP PREPROCESSING}

\subsection{Local Binary Patterns}

Local binary patterns (LBP) were proposed in [5] and have proved to be useful in a variety of pattern recognition tasks. The basic idea is illustrated in Fig. 2: each $3 \times 3$ neighborhood block in the image is thresholded by the value of its center pixel. The 8 results form a binary sequence, representing the pattern at the center point. A decimal representation is obtained by taking the binary sequence as a binary number between 0 and 255. For a pixel, LBP only accounts for its relative relationship with its neighbours, while discarding the information of amplitude, and this makes the resulting LBP values very insensitive to illumination intensities.

The 8-bit binary series with binomial weights consequently result in 256 different patterns in total for the pixel representation. In the initial work of face recognition using LBP [16], a histogram of the LBPs is further calculated, representing the distribution of 256 patterns across the face image.

The advantage of LBP is twofold. Firstly it is a local measure, so LBP in a certain region will not be affected by the illumination conditions in other regions. Secondly it is a relative measure, and is invariant to any monotonic transformation such as shifting, scaling, or logarithm of the pixel-values. Therefore it can be invariant to a certain range of illumination changes.
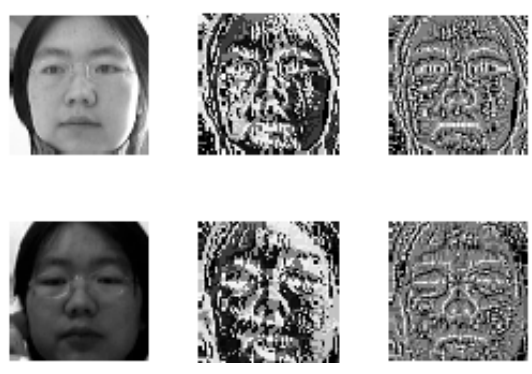

Fig. 3. The effects of LBP preprocessing: first column - the original images under different illuminations; second column - the LBP preprocessed images; third column - simplified LBP preprocessed images. The face size is 64 by 64 .

\subsection{Simplified LBP as a Preprocessing Method}

The distribution of LBPs can be used as a good representation for images with more or less uniform textures, but for the face images it is not enough. A distribution loses connection between the patterns and their relative positions in face. To take advantage of both the local patterns and the positional information, LBP can be used as a preprocessing method, as in [15]. The second column of Fig. 3 shows the effects of LBP preprocessing on the face images.

As shown in Fig. 3, LBP preprocessing acts as a nonlinear high-pass filter on the original image. Therefore, it emphasizes the edges, but as well as the noises. Because noises occur randomly in direction, the exponential weights on the neighbors make the noise effect more prominent. To alleviate this effect, modifications to the original LBP are made in this paper. We assign equal weights to each of the 8 neighborhood, as expressed by

$$
\begin{array}{r}
\operatorname{LBP}(I(x, y))=\sum u\left(I\left(x+\delta_{x}, y+\delta_{y}\right)-I(x, y)\right) \\
\text { where } \quad u(z)= \begin{cases}1 & z>0 \\
0 & z \leq 0\end{cases}
\end{array}
$$

where $\delta_{x}, \delta_{y} \in\{-1,0,1\},\left(\delta_{x}, \delta_{y}\right) \neq(0,0)$. The third column of Fig. 3 shows the resulting face image after the simplified LBP preprocessing. It can be seen that by simplifying the weights, the image becomes more robust to illumination changes. There are two reasons accounting for this phenomenon. Firstly, the simplified LBP preprocessing is not directional-sensitive; secondly the number of total local patterns are largely reduced so the image has a more constrained value domain.

It can be argued that the robustness is obtained at the expense of losing amplitude, and that the simplification of LBP risks the danger of harming discrimination. To tackle the difficult illumination problems by a simple method, these compromises need to be made, and the method remains effective as long as the loss can be surpassed by the gain. Section 5 
will show that this simplified LBP preprocessing method can achieve very good verification performance, with respect to both discrimination and generalization.

\section{VERIFICATION BASED ON LIKELIHOOD RATIO}

Face verification can be seen as a one-class problem [17] where only the user is considered; or a two-class problem if the impostor or background set is also taken into account. The classifier can be distribution based, like Bayesian [18] or likelihood ratio [19], or be boundary based, like SVM (support vector machine) [20]. Given a large and representative sample set, the former solution is ideal, as theoretically no other way could achieve lower error.

In the Bayesian method, the priors of the user and the background distribution are needed, but in reality they are hard to estimate or even to guess. The likelihood ratio method avoids the priors, instead it calculates the ratio and then thresholds it according to certain performance criterion such as false accept rate (FAR) or false reject rate (FRR). It can be formally proved that the likelihood ratio criterion is optimal in the Neyman-Pearson sense [21], i.e., when the FAR is fixed, the likelihood ratio criterion minimizes the FRR, and vice versa.

Our application for the MPD makes it possible to collect considerable amount of the user data during enrolment, therefore we adopt the likelihood ratio method. Another advantage of this method is that it is simple and fast for the MPD hardware as only linear calculations are involved. The likelihood ratio criterion can be expressed as

$$
L(x)=\frac{p_{\text {user }}(x)}{p_{\text {bg }}(x)}>\text { threshold }
$$

where $p_{\text {user }}$ is the user distribution, and $p_{\text {bg }}$ is the background distribution. We assume that both the user class and the background class have a multivariate Gaussian distribution, as expressed by

$$
p(x)=\frac{1}{\sqrt{(2 \pi)^{d}|\Sigma|}} \exp \left(-\frac{(x-\mu)^{T} \Sigma^{-1}(x-\mu)}{2}\right)
$$

where $x$ is the feature vector, $d$ is its dimensionality, $\mu$ is the class mean, and $\Sigma$ is the class covariance. Introducing logarithm, the criterion in (2) can be rewritten

$$
\begin{array}{r}
\ln [L(x)]=\ln \left[p_{\mathrm{user}}(x)\right]-\ln \left[p_{\mathrm{bg}}(x)\right] \\
=\frac{1}{2}\left(\ln \left|\Sigma_{\mathrm{bg}}\right|+\left(x-\mu_{\mathrm{bg}}\right)^{T} \Sigma_{\mathrm{bg}}^{-1}\left(x-\mu_{\mathrm{bg}}\right)\right) \\
-\frac{1}{2}\left(\ln \left|\Sigma_{\mathrm{user}}\right|+\left(x-\mu_{\mathrm{user}}\right)^{T} \Sigma_{\mathrm{user}}^{-1}\left(x-\mu_{\mathrm{user}}\right)\right) \\
>\text { threshold }
\end{array}
$$

where $\mu_{\mathrm{user}}, \mu_{\mathrm{bg}}, \Sigma_{\mathrm{user}}, \Sigma_{\mathrm{bg}}$ are the means and covariances of the user class and background class, respectively. As (4) shows, the logarithm essentially reduces the probability measure to the difference between the two squared Mahalanobis distances in the user and the background class. Fig. 5 will show these two squared distances in a scatter plot, which provide some insight into the discrimination and generalization abilities of the face verification system based on the simplified LBP preprocessing and likelihood ratio classifier.

\section{EXPERIMENTS AND RESULTS}

\subsection{Experiment Setup}

For the face verification system on the MPD, we have adopted efficient algorithms in each stage. Face detection is done by the Viola-Jones detection method [22], and face registration is by aligning the 13 facial landmarks, which are also detected by the Viola-Jones detectors trained by us [23]. All the faces are then scaled to a size of $36 \times 36$ as an empirical choice, as smaller size begin to harm the discrimination, and larger size is not only more costly but also more difficult to generalize. A face ROI is chosen as the upper face region around eyes and nose excluding the mouth to make the feature vectors expression-robust. After this ROI masking and the simplified LBP preprocessing, the reduced feature vector has a length of 440.

For the background data set in (2) we adopt three public face databases, namely the BioID database [24], FERET database [25], and FRGC database [26]. The background data remains the same in all tests.

\subsection{Results on Laboratory Data}

Because the classifier is distribution based, a large number of training data (in our settings at least larger than 440) from a single user are needed. Most public databases, however, do not contain enough data for one user, and thus easily lead to overtraining. Moreover, user data of different sessions are also needed to test the generalization. For these reasons, data collection are set up in laboratory conditions for the purpose of validation.

For each subject, around 1,000 frames of face data are collected, in independent sessions with different illumination conditions. 10 subjects are involved in the data collection. (A larger database is under construction, and the data used in this paper is available from the first author on request.)

We distinguish between two types of test: discrimination and generalization. The first type is closely related to the security aspect of the system, and the second type is closely related to the convenience aspect [1]. Discrimination can be tested on different subjects under the same illumination, as shown in Fig. 4 (a), while generalization can be tested on the same subject under different illumination, as shown in Fig. 4 (b).

As an example, Fig. 5 shows the results of these two tests. We visualize the results in a two dimensional scatter plot, with 


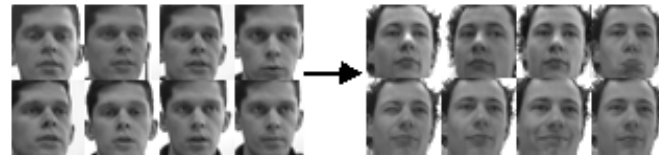

(a) Discrimination test

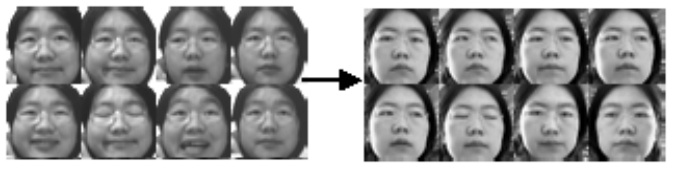

(b) Generalization test

Fig. 4. Two types of test: discrimination and generalization.

the two dimensions indicating the squared Mahalanobis distances in the user and the background space, respectively. In both figures, the circles $\circ$ denote the user training data, the dots - denote the user testing data, the stars $*$ denote the background data, the crosses + denote the tester data, and the line denotes the decision boundary. According to (4), the decision boundary is a straight line with a slope of 1 . Such a twodimensional plot gives a clearer view of the distribution of the user data, background data, and impostor data. Furthermore, it also indicates that the likelihood ratio method involving two opposite classes is superior than the one-class method involving only the user class. This can be observed by comparing the distributions along the two-dimensional space and along the one-dimensional space.

It can be observed from the two examples that both the discrimination and generalization requirements are very well satisfied. Closer observation of (a) and (b) reveals that the user spaces for different users differ vastly, by comparing the same background data distribution in the different user spaces on the horizontal axis. Therefore this user-specific space described by $\mu_{\text {user }}$ and $\Sigma_{\text {user }}$ is able to give a better description of the user, compared to a general intra-personal space sharing covariance between users [18].

Including the data of all sessions, Fig. 6 indicates the overall performance of the face verification system for one user. The classifier is trained by the user data of one session, and then tested on the user data of all the other sessions and on all the impostor data. By adapting the threshold, a receiver operation characteristic (ROC) and equal error rate (EER) can be obtained. We further compare the three different preprocessing methods, namely, high-pass filtering, original LBP, and simplified LBP preprocessing. It can be seen that the simplified LBP preprocessing method outperforms the other two methods. The good performances indicate that the proposed simplification is overall effective, i.e the loss it introduces is surpassed by the gain it brings.

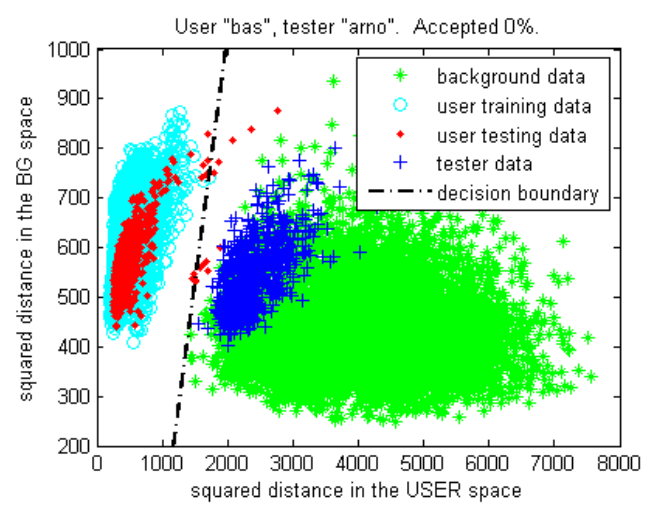

(a) Scatter plot of discrimination test

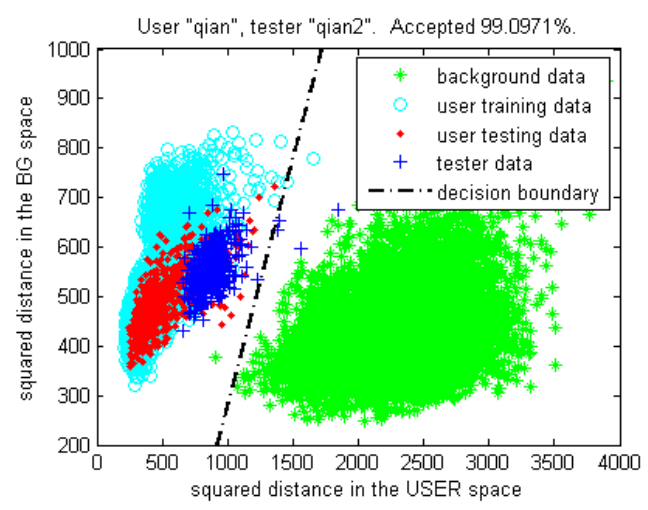

(b) Scatter plot of generalization test

Fig. 5. Scatter plots of the discrimination and generalization tests.

\subsection{Results on the Yale Database $B$}

The algorithm has also been tested on the Yale database B [2], which contains the images of 10 subject, each seen under 576 viewing conditions ( 9 poses $\times 64$ illuminations). Examples of Yale database B and the effects of three preprocessing methods are shown In Fig. 7.

For each subject, the user data are randomly partitioned into $80 \%$ for training, and $20 \%$ for testing. The data of the other 9 subjects are used as the impostor data. ROC can be obtained by thresholding the likelihood ratios of the user test data and the impostor data, and an EER can be obtained as a measure of the performance.

The random partition process is carried out 20 rounds for each subject. We obtain an average and standard deviation of the EER for each of the 10 subjects. The performances of the three different preprocessing methods are compared, as shown in Fig. 8.

It can be seen from Fig. 8 that for all the subjects in the Yale database B, the simplified LBP preprocessing consistently achieves the best performance. This indicates that the simplified LBP preprocessing has higher robustness to large illumination variability compared to the other two methods. 

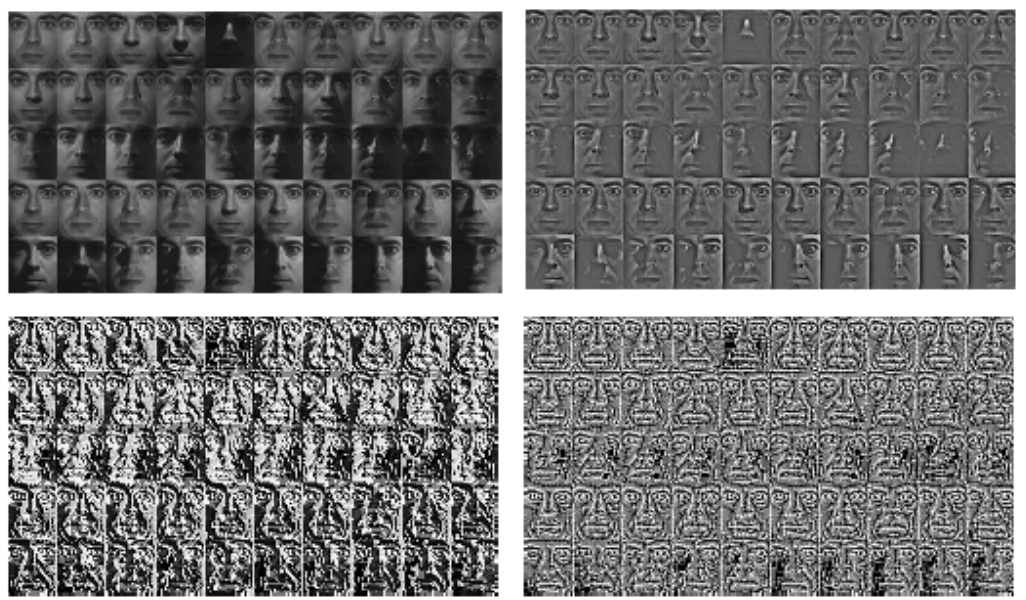

Fig. 7. Examples in Yale database B: left above - the original face images [2]; right above - preprocessed by linear high-pass filter, left below - preprocessed by the LBP with 256 patterns [15]; right below - preprocessed by the simplified LBP.

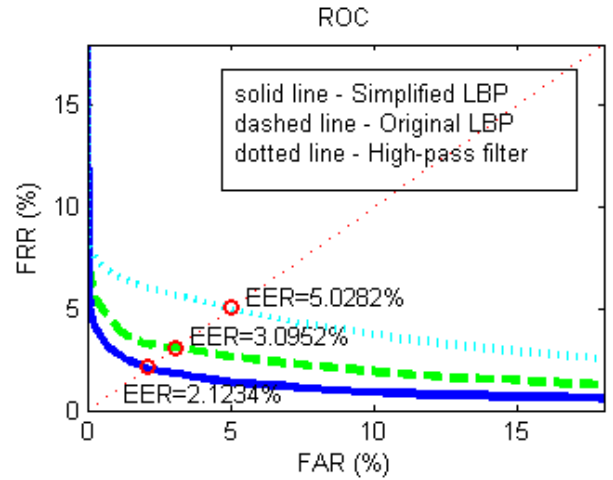

Fig. 6. Verification performance of three different preprocessing methods for one subject in laboratory data.

\subsection{Implementation on MPD}

The efficiency and simplicity of both the feature extraction and verification enables realistic implementation of this system on a MPD. The whole algorithm has been transformed to the Windows Mobile 5 platform in C language onto an Eten M600 mobile device. Without optimization, it takes around 9 seconds to process an image and make an accept-or-reject decision. Profiling indicates that most of the time is spent on the face detection and registration, and that the preprocessing and likelihood-ratio verification are very fast. This system will become practical in use with further optimization both in hardware and software.

\section{CONCLUSIONS}

Discrimination ability and generalization ability are two equally important aspects in face verification system. We propose a

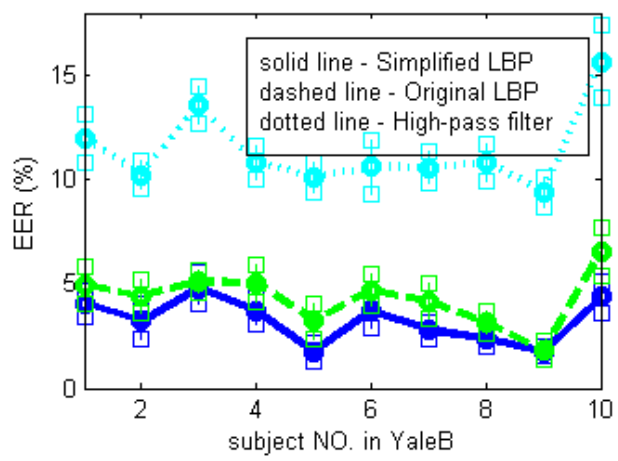

Fig. 8. Verification performance of three different preprocessing methods for 10 subjects in Yale database B.

simplified implementation of LBP as a preprocessing method for face verification. The simplification of the original LBP increases the generalization for the user under different illuminations, at little expenses of discrimination against imposters. This method is very simple and fast for hardware implementation. In combination with the theoretically optimal likelihood-ratio classifier, the face verification system achieves very good performance with respect to both discrimination and generalization, with an EER of around $2 \%$ in our face verification system on MPD.

\section{REFERENCES}

[1] Q. Tao and R. Veldhuis, "Biometric authentication for mobile personal device," in First International Workshop on Personalized Networks, San Jose, USA, 2006.

[2] A. Georghiades, P. Belhumeur, and D. Kriegman, "From 
few to many: Illumination cone models for face recognition under variable lighting and pose," IEEE Transactions on Pattern Analysis and Machine Intelligence, vol. 23, no. 6, pp. 643-660, 2001.

[3] Y. Moses, Y. Adini, and S. Ullman, "Face recognition: The problem of compensating for changes in illumination direction," in In European Conference on Computer Vision, 1994.

[4] P. Belhumeur, J. Hespanha, and D. Kriegman, "Eigenfaces vs. fisherfaces: Recognition using class specific linear projection," IEEE Transactions on Pattern Analysis and Machine Intelligence, vol. 19, no. 7, pp. 711$720,1997$.

[5] T. Ojala, M. Pietikainen, and T. Maenpaa, "Multiresolution gray-scale and rotation invariant texture classification with local binary patterns," IEEE Transactions on Pattern Analysis and Machine Intelligence, vol. 24, no. 7, pp. 971-987, 2004.

[6] P. Belhumeur and D. Kriegman, "What is the set of images of an object under all possible illumination conditions," International Journal of Computer Vision, vol. 28, no. 3, pp. 1-16, 1998.

[7] R. Basri and D. Jacobs, "Lambertian reflectances and linear subspaces," in IEEE International Conference on Computer Vision, 2001.

[8] S. Zhou and R. Chellapa, "Illumination light field: image-based face recognition across illumination and pose," in IEEE International Conference on Automatic Face and Gesture Recognition, 2004.

[9] E. Land and J. McCann, "Lightness and retinex theory," Journal of the Optical Society of America, vol. 61, no. 1, pp. 1-11, 1971.

[10] D. Jobson, Z. Rahmann, and G. Woodell, "Properties and performance of a center/surround retinex," IEEE Transactions on Image Processing, vol. 6, no. 3, pp. 451-462, 1997.

[11] A. Shashua and T. Riklin-Raviv, "The quotient image: class based re-rendering and recognition with varying illuminations," IEEE Transactions on Pattern Analysis and Machine Intelligence, vol. 23, no. 2, pp. 129-139, 2001.

[12] H. Wang, S. Li, and Y. Wang, "Face recognition under varying lighting conditions using self quotient image," in IEEE International Conference on Automatic Face and Gesture Recognition, 2004.
[13] P. Perona and J. Malik, "Scale-space and edge detection using anisotropic diffusion," IEEE Transactions on Pattern Analysis and Machine Intelligence, vol. 12, no. 7, pp. 629-639, 1990.

[14] T. Chan, J. Shen, and L. Vese, "Variational pde models in image processing," Notices of the American Mathematical Society, vol. 50, no. 1, pp. 14-26, 2003.

[15] G. Heusch, Y. Rodriguez, and S. Marcel, "Local binary patterns as image preprocessing for face authentication," in IEEE International Conference on Automatic Face and Gesture Recognition, 2006.

[16] T. Ahonen, M. Pietikinen, A. Hadid, and T. Menp, "Face recognition based on the appearance of local regions," in International Conference on Pattern Recognition, 2004.

[17] D. Tax, "One class classification," Ph.D. thesis, Delft University of Technology, 2001.

[18] B. Moghaddam, T. Jebara, and A. Pentland, "Bayesian face recognition," Bayesian face recognition, vol. 33, no. 11, pp. 771-782, 2000.

[19] R. Veldhuis, A. Bazen, J. Kauffman, and P. Hartel, "Biometric verification based on grip-pattern recognition," in Proceedings of SPIE, 2004.

[20] N. Cristianini and J. Shawe-Taylor, An Introduction to Support Vector Machines and other kernel-based learning methods, Cambridge University Press, 2000.

[21] H.L. van Trees, Detectioin, Estimation, and Modulation Theory, John Wiley and Sons, New York, 1969.

[22] P. Viola and M. Jones, "Robust real-time face detection.," International Journal of Computer Vision, vol. 57, no. 2, pp. 137-154, 2004.

[23] G. M. Beumer, Q. Tao, A. M. Bazen, and R. N. J. Veldhuis, "A landmark paper in face recognition," in IEEE International Conference on Automatic Face and Gesture Recognition, 2006.

[24] HumanScan, "Bioid face database," http://www . humanscan.de/.

[25] NIST, "Feret face database," http://www.itl. nist.gov/iad/humanid/feret/.

[26] NIST, "Frgc face database," http://face.nist. gov/frgc/. 